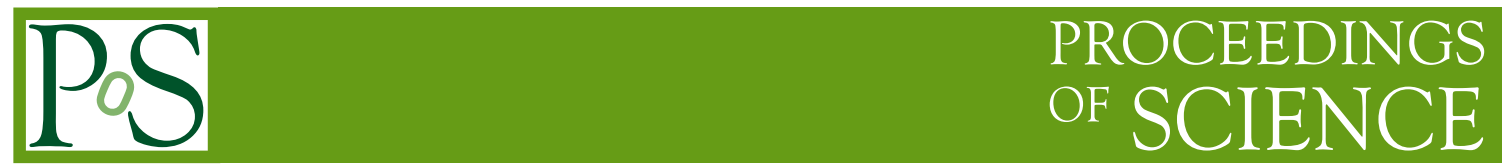

\title{
Planar sensors for future vertex and tracking detectors
}

\author{
Alexander Dierlamm*t \\ Institut für Experimentelle Kernphysik, Karlsruhe Institute of Technology \\ E-mail: alexander.dierlammekit.edu
}

\begin{abstract}
With increasing instantaneous and integrated luminosities of collision experiments the requirements on position sensitive detectors and sensor materials increase rapidly. Numerous R\&D projects of several collaborations have investigated known and new materials with respect to their range of application.

This report gives an overview of the current activities and common understanding of planar sensors concentrating on operational aspects.
\end{abstract}

22nd International Workshop on Vertex Detectors,

15-20 September 2013

Lake Starnberg, Germany

*Speaker.

$\dagger$ on behalf of the CMS Tracker Collaboration 


\section{Introduction}

Planar silicon sensors have proven reliable operation in tracking and vertex detectors of current and former HEP experiments [1, 2, 3, 4] and are therefore considered as baseline for future detectors. These are mainly the upgraded Trackers of the LHC experiments for the high luminosity phase (HL-LHC) [5], which is planned to start in 2023. The radiation environment for the silicon sensors will be a factor of about six higher than the design goal for the currently operated sensors. Therefore the collaborations have been investigating silicon materials and technologies to find more radiation tolerant solutions $[6,7,8]$. The main outcome was that electron read-out is superior to hole read-out, which is the read-out mode for p-in-n sensors used in the current tracking detectors. To avoid the double-sided process of $n$-in-n sensors (higher costs and lower yield than single-sided process), $\mathrm{RD}^{1} 0^{1}$ established the use of $\mathrm{n}$-in-p sensors, which have been in the focus of research in the last few years.

It was also found in [9] that sensors thinner than the nominal $300 \mu \mathrm{m}$ collect more charge than expected after high irradiation $\left(\sim 3 \cdot 10^{15} \mathrm{n}_{\mathrm{eq}} \mathrm{cm}^{-2}\right)$ and can eventually exceed the charge collection of $300 \mu \mathrm{m}$ devices at very high fluences $\left(>1 \cdot 10^{16} \mathrm{n}_{\mathrm{eq}} \mathrm{cm}^{-2}\right)$. Since thinner sensors also contribute less material to the detector the option to use $200 \mu \mathrm{m}$ sensors is pursued both in ATLAS and CMS Trackers and even thinner materials are discussed for the pixel vertex detectors. Thinner sensors have less trapping due to the faster drift of the charge carriers in the higher electric fields at the same bias voltage. In addition, less current is generated in the smaller bulk volume up to very high fluences $\left(\sim 1 \cdot 10^{16} \mathrm{n}_{\mathrm{eq}} \mathrm{cm}^{-2}\right)$ if no charge multiplication is present. The drawback is a reduction of generated charge carriers at low irradiation fluences compared to $300 \mu \mathrm{m}$ thick sensors. See also [10] in this issue.

In some cases, charge multiplication (CM) effects occur after strong irradiation $\left(>3 \cdot 10^{15} \mathrm{n}_{\mathrm{eq}} \mathrm{cm}^{-2}\right)$ close to the strip implants due to the very high fields and large charge carrier densities, which strongly increase the impact ionization rate. This CM does not only amplify the signal, but also exhibits an increase of the leakage current, which increases the noise in the read-out system. So instead of an increased signal to noise ratio, there might be just the unwanted increase of power dissipation.

Some applications of strip sensors require special routing lines to transfer the signal from the strip implants to the contact pads. The metal routing lines can be implemented in the first or second metal layer. Undesired coupling to routing lines can occur for both implementations as will be shown in Section 3.

When using electron read-out with $\mathrm{n}+$ implants one has to take care of the strip isolation since the positive oxide charge generated by ionizing radiation leads to an accumulation of electrons which could cause the strips to short electrically. New measurements from a CMS campaign and simulations indicate an interplay of bulk and surface defects, which allow a moderate $\mathrm{p}$-stop concentration to guarantee good strip isolation up to $1 \cdot 10^{16} \mathrm{n}_{\mathrm{eq}} \mathrm{cm}^{-2}[11,12]$.

p-type sensors on the one hand are easier and cheaper to produce, but on the other hand have the disadvantage that the front edge is on the same high potential as the backside. This is problematic especially for pixel sensors ${ }^{2}$, which are facing the read-out chip at a very small distance and are

\footnotetext{
${ }^{1}$ http://rd50.web.cern.ch/rd50/

${ }^{2}$ Similar problems can occur for strip sensors and flat wire bonds.
} 


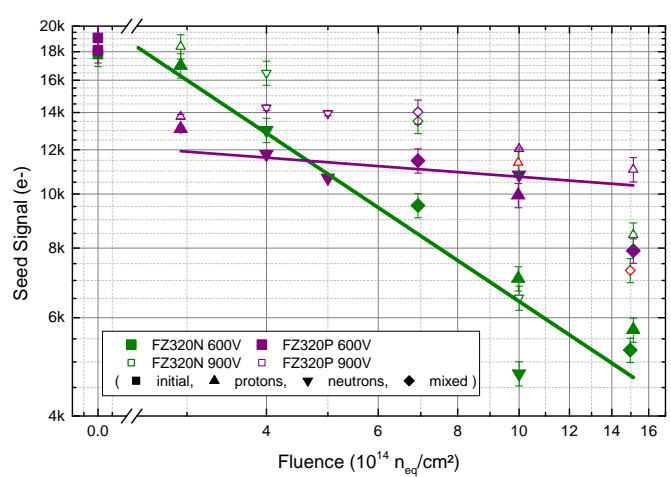

(a) Charge collection vs. fluence for $300 \mu \mathrm{m}$ thick FZ sensors at $-20^{\circ} \mathrm{C}$.

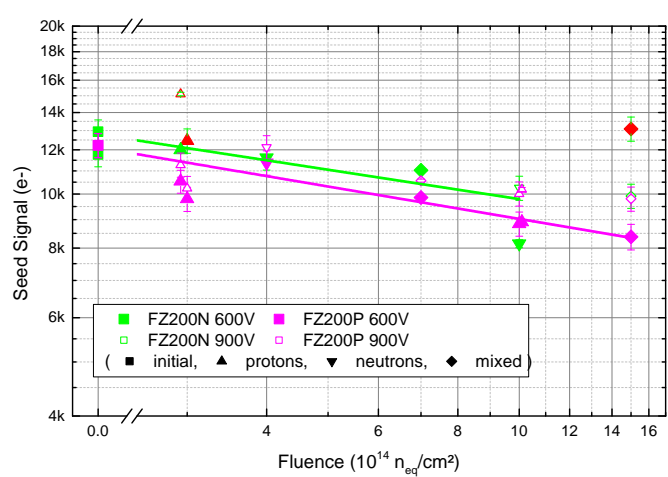

(b) Charge collection vs. fluence for $200 \mu \mathrm{m}$ thick FZ sensors at $-20^{\circ} \mathrm{C}$.

Figure 1: The charge collection of sensors with electron read-out is superior to hole read-out above 7 . $10^{14} \mathrm{n}_{\mathrm{eq}} \mathrm{cm}^{-2}$ for both $320 \mu \mathrm{m}$ and $200 \mu \mathrm{m}$ thick sensors. The red symbols belong to measurements on p-inn type sensors, which showed strong non-Gaussian noise producing random ghost hits (Section 2.2). The different symbol shapes indicate different particle types. Lines are power fits $\left(y=a \cdot x^{b} ; F>0\right)$ to data points at $600 \mathrm{~V}$.

prone to arcing. Several coatings are being investigated [6] as well as a new proposal by Hamamatsu Photonics K.K. to use an $\mathrm{n}^{+}$edge implantation [13].

Considering mass production of silicon sensors one has to limit production costs. One way is to increase the wafer size to 8 inch (mainly 6 inch for strip sensors at the moment), which will reduce the costs per unit area. In case the larger area can be efficiently used, this step could help controlling the budget. Still, the producibility, especially with $200 \mu \mathrm{m}$ thick material, needs to be proven.

\section{Charge collection}

The charge collection of different silicon materials has been investigated by the RD50 collaboration for several years in many laboratories and with several producers. The outcome of this effort are many important results and deep analyses of the microscopic defect formation, and also led to the development of p-type sensors. To confirm these results and extend the aims CMS launched an extensive R\&D campaign with prototype sensors of one vendor (Hamamatsu Photonics K.K.) made from different silicon materials and sensor thicknesses [14].

\subsection{Charge loss}

One of the many aspects investigated in this campaign was the charge collection of p-type vs. n-type sensors in $200 \mu \mathrm{m}$ and $320 \mu \mathrm{m}$ thickness (Figure 1). In these plots the collected charge of the seed strip is plotted, which is a more meaningful parameter for binary read-out as it will be used for the future CMS Strip Tracker. It is obvious that p-in-n sensors suffer from a strong charge loss above $7 \cdot 10^{14} \mathrm{n}_{\mathrm{eq}} \mathrm{cm}^{-2}$, while p-type sensors with electron read-out show a slower decrease of the charge collection, which could be parametrized by an exponential function. The annealing of the seed charge is plotted in Figure 2 for float zone (FZ), magnetic Czochralski (MCz) and deep- 


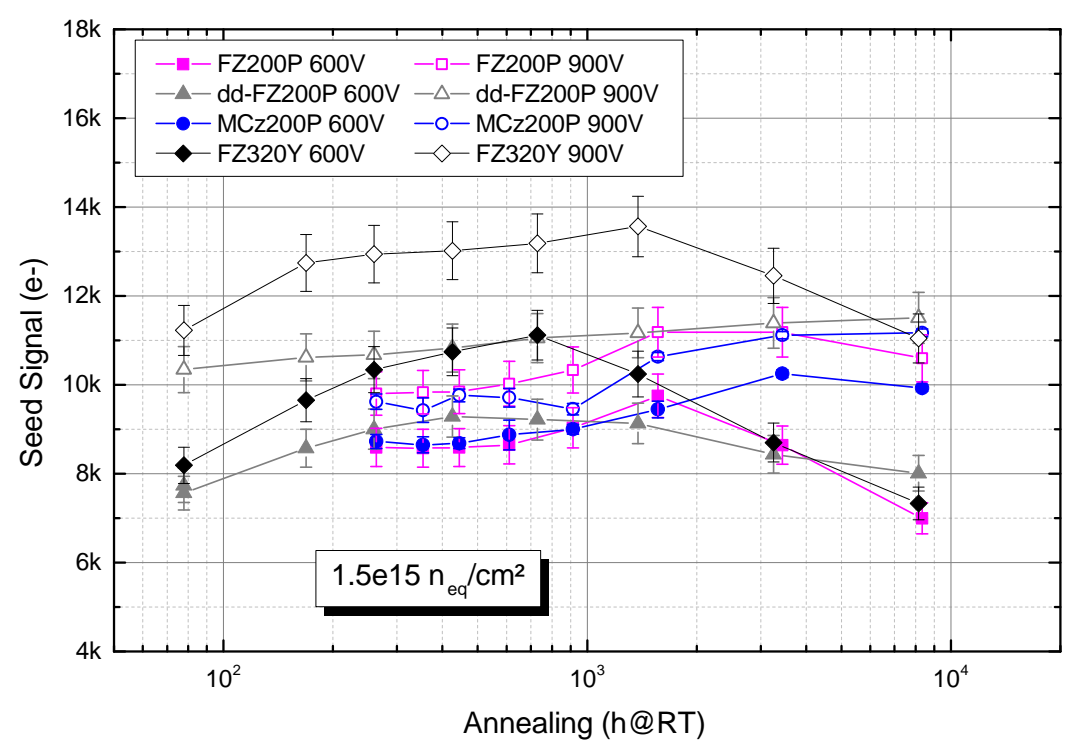

Figure 2: Annealing of the seed signal. The signal is more or less constant over a period of 20 weeks (3360 hours) at room temperature. $\mathrm{MCz}$ material seems to stay constant or even increases for longer annealing times. The annealing was performed at $60 / 80^{\circ} \mathrm{C}$ and the scaling to $21^{\circ} \mathrm{C}$ was done according to the annealing behaviour of the leakage current [15]. FZ320Y stands for $320 \mu$ m thick FZ n-in-p sensors with p-spray strip isolation.

diffused $\mathrm{FZ}^{3}$ (dd-FZ). The thin sensors show a quite constant annealing behaviour, while the thick sensor has a pronounced maximum at $600 \mathrm{~V}$ after the beneficial annealing. The constant signal can be exploited by deliberate heating (or not cooling) of the sensors during maintenance. That would not change the signal, but reduce the leakage current and therefore the noise for a better signal to noise ratio.

\subsection{Non-Gaussian noise}

In addition to the lower charge collection at high fluence, the measurements showed nonGaussian noise for the p-in-n-type sensors after irradiation in some cases (indicated as red symbols in the plots of Figure 1). Figure 3 shows the standard case with a Gaussian noise distribution and one example of a non-Gaussian distribution, which leads to noise charge above the signal thresholds (seed signal $>5 \times \sigma$ ) thus being interpreted as particle hits. These noise hits are equally distributed over the entire sensor and have been named Random Ghost Hits (RGHs). Similar noise features have been reported previously and called micro-discharge or grass noise, but since it is not clear, whether the origin is the same, a new name was introduced. Actually, the temperature dependence is different to the one reported in [16] for micro-discharge: the RGHs are more pronounced with increasing temperature. It was also found that the RGH rate (hits per strip and per event, which is equivalent to a noise occupancy, and should be much less than the particle hit occupancy of 1-2\%) depends on the bias voltage and annealing time (Figure 4(a)). This effect is most pronounced at

\footnotetext{
${ }^{3}$ The active thickness of these $320 \mu \mathrm{m}$ thick sensors was reduced by a deep backside doping to $200 \mu \mathrm{m}$.
} 


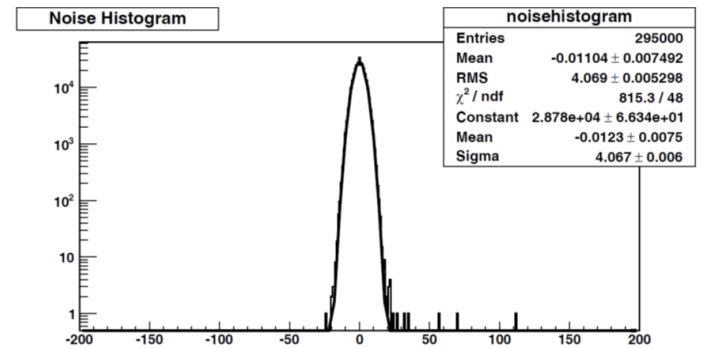

(a) Normal noise distribution.

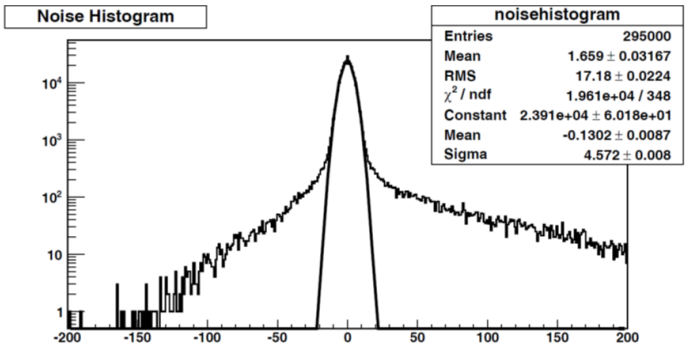

(b) Noise distribution with non-Gaussian tails producing random ghost hits (RGH).

Figure 3: A normal noise distribution has Gaussian shape and a definition for a signal above $5 \times \sigma$ easily distinguishes noise and particle hit. In contrast, the same definition fails for a large faction of events for a non-Gaussian distribution as on the right. Sensors with such a noise behaviour would generate too many fake hits and should therefore not be used.

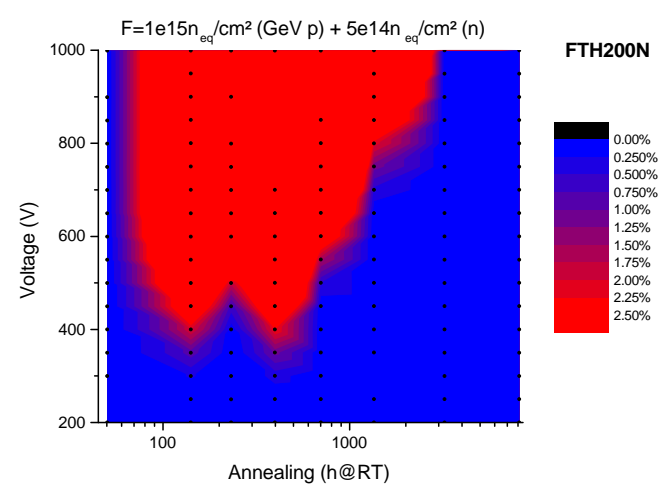

(a) RGH occupancy as a function of annealing and bias voltage. Here the pitch is $80 \mu \mathrm{m}$ and $\mathrm{w} / \mathrm{p}=0.23$.

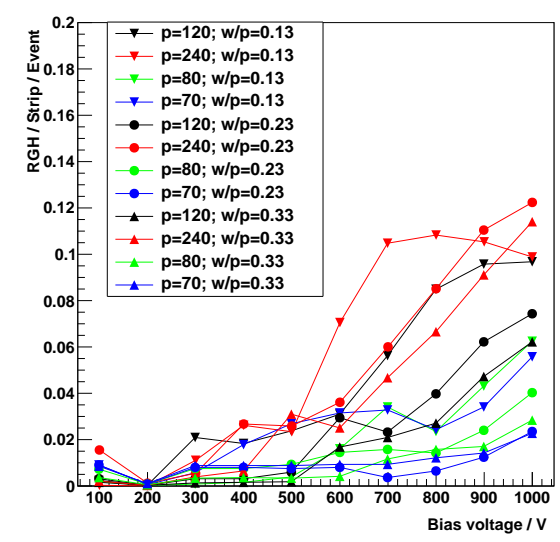

(b) Measured dependence on the strip geometry. RGHs are more pronounced for large pitch and small $\mathrm{w} / \mathrm{p}$ ratio.

Figure 4: Examples for RGHs for a $200 \mu \mathrm{m}$ thick p-in-n FZ strip sensor irradiated to a fluence of $1 \cdot 10^{15} \mathrm{n}_{\mathrm{eq}} \mathrm{cm}^{-2}$ by $23 \mathrm{GeV}$ protons plus $5 \cdot 10^{14} \mathrm{n}_{\mathrm{eq}} \mathrm{cm}^{-2}$ by neutrons.

high bias voltages and after about equivalent ${ }^{4} 100$ to 200 hours at room temperature. The annealing time at which the RGH rate reaches its maximum coincides with a period of reduced full depletion voltage (beneficial annealing), which results in higher electric fields at a constant bias voltage. This effect was observed for $320 \mu \mathrm{m}$ and $200 \mu \mathrm{m}$ thick p-in-n sensors made of FZ or MCz material irradiated with low energy ( $23 \mathrm{MeV}$ from compact cyclotron at $\mathrm{KIT}^{5}$ ) or high energy $(23 \mathrm{GeV}$ from PS at $\mathrm{CERN}^{6}$ ) protons. On a special multi-geometry strip sensor structure the dependence on geometry was investigated. Figure 4(b) shows that for large pitch and small width to pitch ratio (w/p)

\footnotetext{
${ }^{4}$ The annealing was performed at $60 / 80^{\circ} \mathrm{C}$ and the scaling to $21^{\circ} \mathrm{C}$ was done according to the annealing behaviour of the leakage current [15].

${ }^{5}$ http://www.ekp.kit.edu/english/irradiation_center.php

${ }^{6}$ https://irradiation.web.cern.ch/irradiation/irrad1.htm
} 


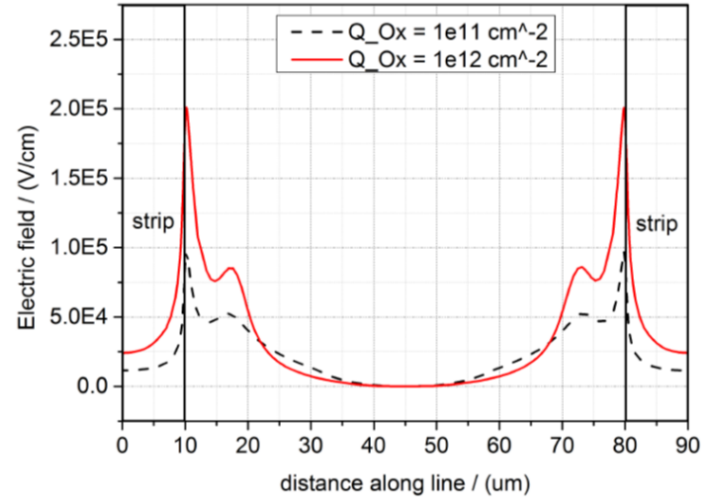

(a) p-in-n strip sensor

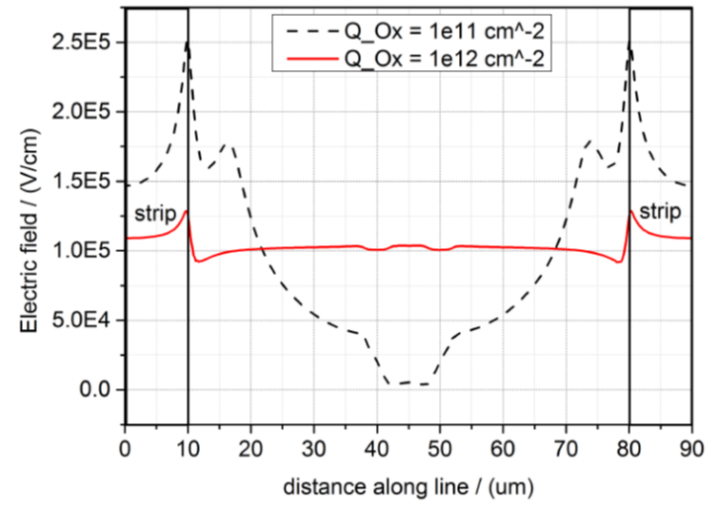

(b) n-in-p strip sensor with p-stop strip isolation

Figure 5: Simulated electric fields at the surface of a strip sensor after a proton fluence of $1 \cdot 10^{15} \mathrm{n}_{\mathrm{eq}} \mathrm{cm}^{-2}$ at $1000 \mathrm{~V}$ and $-20^{\circ} \mathrm{C}$. Bulk damage was introduced by a two trap model listed in Table 1 . Shown is the region between two strips. The electric field strength tends to increase with $\mathrm{Q}_{\mathrm{ox}}$ for $\mathrm{p}$-in-n sensors, while for $\mathrm{n}$-in- $\mathrm{p}$ sensors it drops.

\begin{tabular}{cccc}
\hline Parameter & Unit & Donor & Acceptor \\
\hline Energy & $\mathrm{eV}$ & $\mathrm{E}_{\mathrm{V}}+0.48$ & $\mathrm{E}_{\mathrm{C}}-0.525$ \\
Concentration & $\mathrm{cm}^{-3}$ & $5.598 \mathrm{~cm}^{-1} \times \mathrm{F}-3.949 \cdot 10^{14}$ & $1.189 \mathrm{~cm}^{-1} \times \mathrm{F}-6.454 \cdot 10^{13}$ \\
$\sigma(\mathrm{e})$ & $\mathrm{cm}^{2}$ & $1.0 \times 10^{-14}$ & $1.0 \times 10^{-14}$ \\
$\sigma(\mathrm{h})$ & $\mathrm{cm}^{2}$ & $1.0 \times 10^{-14}$ & $1.0 \times 10^{-14}$ \\
\hline
\end{tabular}

Table 1: Parameters used for bulk defects in the T-CAD simulation [11], where $\sigma(\mathrm{e} / \mathrm{h})$ are the electron and hole cross sections, respectively, and $\mathrm{F}$ is the irradiation fluence.

the noise hits are more pronounced.

Neutron irradiated sensors showed a reduced RGH rate compared to sensors at similar proton fluences. This indicates a relation to surface damage, especially charge up of the silicon oxide due to ionizing radiation, which is much smaller for neutron irradiation (still there is some gamma radiation component in the radiation field of the used reactor at $\left.\operatorname{JSI}^{7}[17,18]\right)$.

It is not easy to spot this behaviour without systematic scan of the phase space. Therefore possible measurements of such effects on other sensors than the ones described above might just have been reported as 'noisy'.

Complementing TCAD simulations with Synopsys Sentaurus ${ }^{8}$ showed an interesting intrinsic difference of $\mathrm{p}$-in-n and n-in-p sensors after irradiation (to simulate the bulk damage two effective traps have been introduced with parameters listed in Table 1): while electric fields at the strip edges in p-in-n sensors increase with increasing oxide charge $\mathrm{Q}_{\mathrm{ox}}$ (Figure 5(a)), which represents higher irradiation with ionizing particles, the n-in-p sensors in contrast show lower electric field maxima with increasing $\mathrm{Q}_{\mathrm{ox}}$. In a simplistic view the positive surface charge between the strips does repel the holes and limits the low potential to the strips resulting in higher electric fields there. In

\footnotetext{
${ }^{7}$ http://www.rcp.ijs.si/ric/description-a.html

${ }^{8}$ http://www.synopsys.com/Tools/TCAD/DeviceSimulation
} 


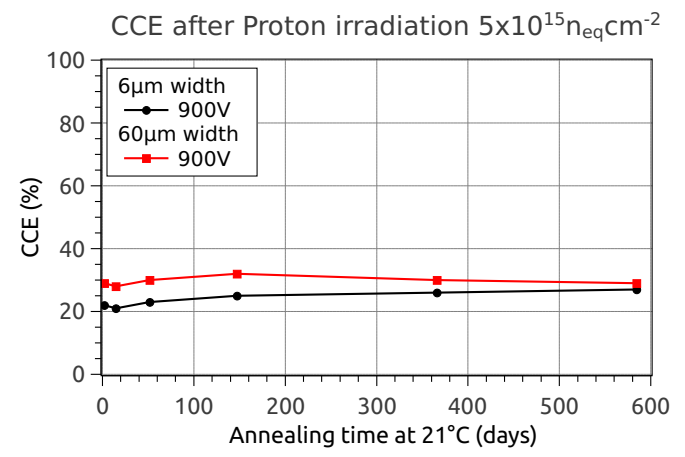

(a) After proton irradiation

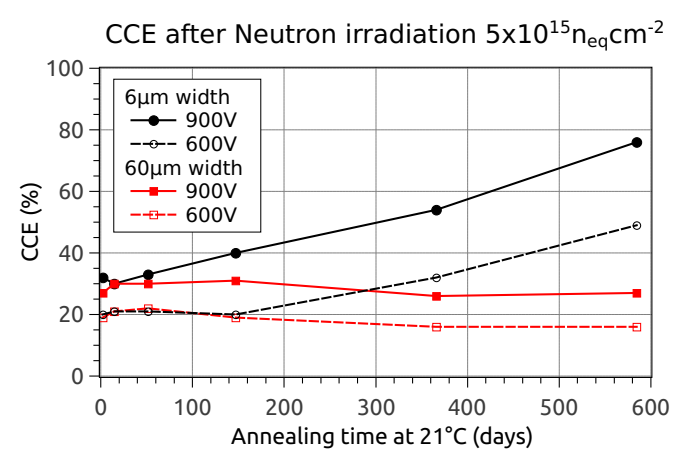

(b) After neutron irradiation

Figure 6: Charge collection efficiency for irradiated n-in-p strip sensors with a pitch of $80 \mu \mathrm{m}$ and $w / p$ of 0.075 and 0.75 . Only the neutron irradiated sensor shows a significant increase in CCE due to CM. The annealing was accelerated by elevated temperature and the time scaled to room temperature according to the scaling of leakage current. [20]

contrast, for p-type, the electrons are attracted by the positive surface charge and the low potential area is increased resulting in lower electric field maxima. These results confirm the experimental observations, that $n$-in-p sensors are more robust at high irradiations, since the electric fields are not as high as in the p-in-n sensors and therefore are less prone to break-down, micro-discharge or other noise effects resulting from high electric fields.

\subsection{Charge multiplication}

In a dedicated study of the RD50 collaboration a set of n-in-p type strip sensors was designed with varying strip pitch $(40 \mu \mathrm{m}-100 \mu \mathrm{m})$, w/p $(0.075-0.75)$, thickness $(150 \mu \mathrm{m}-675 \mu \mathrm{m})$ and implant shape for the $\mathrm{n}^{+}$-electrodes (standard, double diffusion time, double ion energy) [19, 20]. After the production at Micron Semiconductor Ltd. the samples have been irradiated with protons at KIT and neutrons at JSI. This study showed no CM up to $1 \cdot 10^{15} \mathrm{n}_{\mathrm{eq}} \mathrm{cm}^{-2}$ for the investigated samples, while for a neutron fluence of $5 \cdot 10^{15} \mathrm{n}_{\mathrm{eq}} \mathrm{cm}^{-2} \mathrm{CM}$ has been observed for sensors with small $\mathrm{w} / \mathrm{p}$ and double ion energy (the doubly charged ions have a smaller penetration depth resulting in a shallow implant) just after irradiation.

In [20] the annealing behaviour has been investigated and it was observed that sensors with small w/p show increasing CM with annealing time after neutron irradiation, while proton irradiated samples do not show this effect (Figure 6). This is again a strong indication that surface damage can alter the electric field configuration at the strips and therefore influence significantly the charge collection behaviour.

Further approaches to stimulate CM are discussed in [10].

Still, the increased charge collection comes with an increase in leakage current and the increase of noise has to be evaluated with the foreseen read-out electronic. The test system used in [20] showed a doubling of the noise during the annealing period shown in 6(b) for $6 \mu \mathrm{m}$ wide strips at $900 \mathrm{~V}$. Only if one can profit from an increase in S/N one can consider making use of this kind of operation mode taking into account the required cooling for the additional dissipated power. 


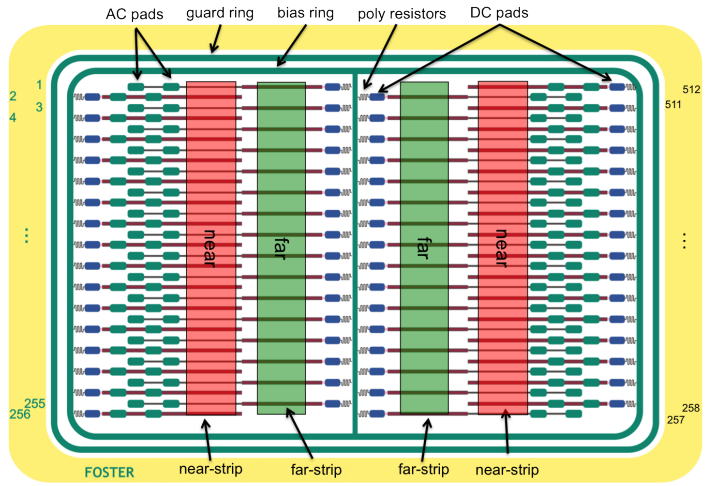

(a) Sketch of a FOSTER. The far strips in the centre are connected to the contact pads at the edge of the sensor by narrow routing lines in the first metal layer.

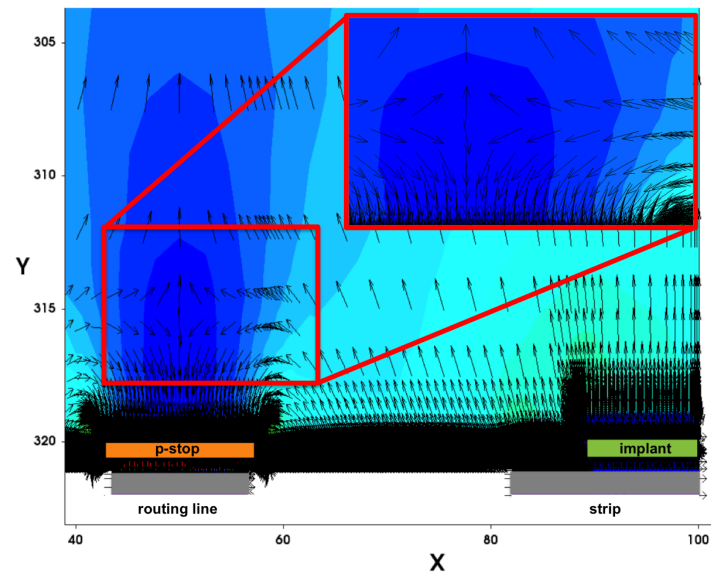

(b) Simulation of the electric field in case a p-stop layer is placed below the routing line. The field is inflected just below the p-stop and the electrons are prevented from reaching the surface and inducing signal in the routing line, which would spoil the identification of the particle hit. [22]

Figure 7: The FOSTER concept can increase the granularity by keeping the advantage of read-out contacts at the sensor edge. Initial versions with routing-lines above undoped silicon showed unwanted coupling effects, which could be mitigated by a p-doped layer below.

\section{Signal coupling with routing lines}

As mentioned in the introduction, some applications of strip sensors require special on-sensor routings to the read-out electronics. One example are the silicon sensors for the VELO detector, which have routing lines in a second metal layer separated from the first metal layer by about $1 \mu \mathrm{m}$ silicon oxide. This was considered a robust technique to transmit signals without influencing neighbouring strips or routing lines. Still, after irradiation parasitic coupling effects appeared, which led to some (still tolerable) cluster finding inefficiency [21]. Observations indicate that the effect saturates or even improves with further irradiation. This effect has not been understood yet, but is not a limiting factor for the upgrade, since the VELO collaboration decided to use pixellated sensors, which do not need routing.

Another application for the use of routing lines is the so called FOSTER ${ }^{9}$, which implements a strip sensor with four strip segments, which all can be read out at the sensor edge [22, 23]. This allows a higher granularity by keeping the possibility to use these sensors for the CMS $\mathrm{p}_{\mathrm{T}}$-module concept, for which two stacked sensors are read-out by one hybrid at the edge [24]. Figure 7(a) shows a drawing of a FOSTER. Initial prototypes from the CMS campaign showed unwanted signals on far-strips (connected to the contact pads via routing lines), when charge carriers were injected in the near region close to the routing lines. T-CAD simulations helped to find a cure for this problem. In p-type devices one can implement the necessary p-stop strip isolation in such a way that the pstop area is just below the routing line. In that configuration the electric field lines are inflected below the p-stop (Figure 7(b)) and the contribution of the induced electron signal is much reduced.

\footnotetext{
${ }^{9}$ FOur-fould segemented STrip sensor with Edge Read-out
} 
Prototypes with the suggested p-doped layer below the routing lines have been produced by IMB$\mathrm{CNM}^{10}$ and showed the expected good performance. The properties after irradiation are still under investigation.

\section{Processing issues}

The charge collection is mainly driven by the choice of bulk material and read-out polarity, but can also be influenced by the way the strips are implemented as discussed in Section 2.3. There are many more aspects to be considered when processing the sensors (mask layout, doping concentrations, passivation,...) and one of them is chosen for further discussion here.

\subsection{Inter-strip resistance}

Sensors with electron read-out ( $\mathrm{n}$-in-n and n-in-p type) need some precaution to isolate the $\mathrm{n}^{+}$strips from one another. This is due to the always slightly positive charged oxide, which attracts electrons. The electron accumulation layer would lower the inter-strip resistance significantly and short all strips, which needs to be prevented. Several options are available like a moderately pdoped layer (p-spray) or highly p-doped structures surrounding the strips in different geometries (p-stop). Figure 8(a) shows measurement results obtained in a CMS campaign for the inter-strip resistance versus irradiation fluence, which demonstrates sufficient ${ }^{11}$ isolation for the p-spray and p-stop solution. This result is especially interesting since the doping concentrations have been measured and estimated to be quite low (peak concentrations: [p-spray] $\sim 1 \cdot 10^{15} \mathrm{~cm}^{-3}$, [p-stop] $5 \cdot 10^{15} \mathrm{~cm}^{-3}$ ) [25]. T-CAD simulations could not reproduce the good isolation properties with the low doping concentrations and a surface charge density of $5 \cdot 10^{11} \mathrm{~cm}^{-2}$ expected for a fluence of $5 \cdot 10^{14} \mathrm{n}_{\mathrm{eq}} \mathrm{cm}^{-2}$. But as soon as the bulk damage is modelled by adding defects corresponding to increasing fluence, the isolation is established (Figure $8(\mathrm{~b})$ ) due to a space charge region formed by ionized traps surrounding the strips.

\section{Summary and outlook}

Many studies have shown that electrons read-out is superior to holes read-out at high radiation environments concerning signal charge and high field effects. With the development of n-in-p-type sensors one can now reduce the production costs and gain robustness in handling compared to $\mathrm{n}$-in-n devices. Further understanding of the inter-strip isolation allows the production of robust sensors and can also be used to mitigate coupling effects to routing lines. Both ATLAS and CMS have chosen p-type strip sensors as baseline for their tracking detectors and are evaluating possible vendors for the large scale productions.

p-type planar sensors would be a good option for pixel vertex detectors if a stable process for large scale production is identified to protect the closely spaced read-out chips from HV sparking. In general at very high fluences, thin sensors are preferred and together with low thresholds of the future read-out electronics the good resolution can be maintained. Also for the assembly of thin

\footnotetext{
${ }^{10}$ Instituto de Microelectronica de Barcelona, IMB-CNM-CSIC, Barcelona, Spain

${ }^{11}$ inter-strip resistance should be at least $10 \times$ larger than the bias resistance
} 


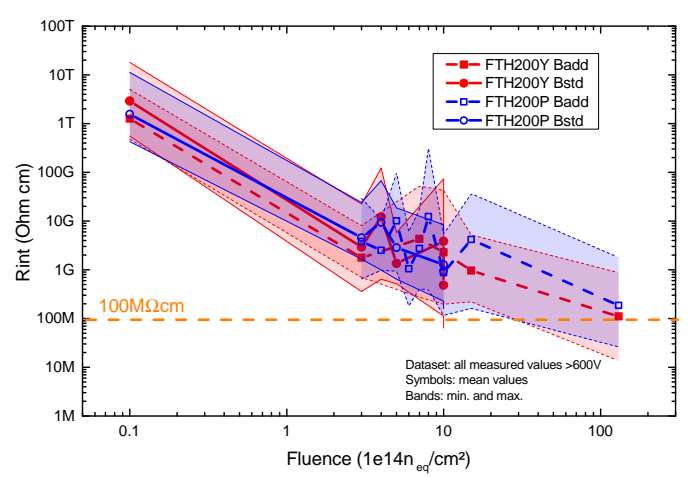

(a) Inter-strip resistance as a function of fluence. Badd and Bstd are two sensor types, which just differ in number of strips and strip length. A good $R_{\text {int }}$ should be much larger than the bias resistance of about $2 \mathrm{M} \Omega$. $100 \mathrm{M} \Omega \mathrm{cm}$ results in $20 \mathrm{M} \Omega$ for a $5 \mathrm{~cm}$ long strip, which is the baseline for a CMS strip sensor.

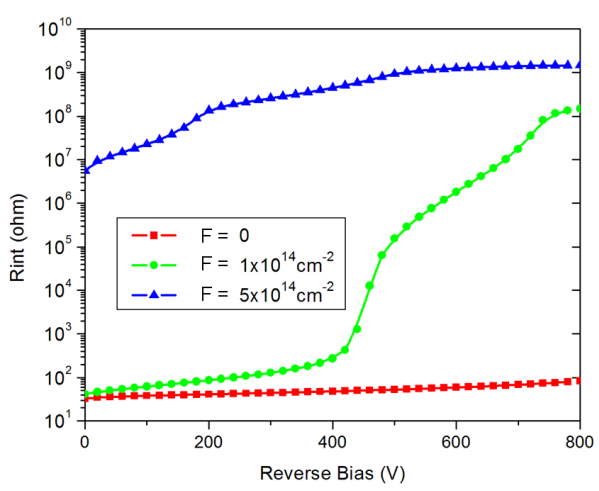

(b) Simulation of the inter-strip resistance in presence of surface oxide charge $\left(5 \cdot 10^{11} \mathrm{~cm}^{-2}\right)$ and increasing bulk damage [12]. Without bulk damage the moderate peak p-stop concentration of $5 \cdot 10^{15} \mathrm{~cm}^{-3}$ is not sufficient to isolate the strips. With increasing bulk damage the isolation is established again.

Figure 8: Inter-strip resistance measurements within the CMS campaign on HPK samples showed high values even at very high fluences. This was surprising given the quite low doping concentration of the isolating structure. TCAD simulations can only reproduce the good isolation, if bulk damage is taken into account.

sensors and read-out chips a reliable process needs to be established with industry.

Planar sensors can be used up to very high fluences and in a large fraction of pixel vertex detectors. The future read-out chips will show where the transition to more complex sensor technologies ( $3 \mathrm{~d}$ silicon sensors, diamond sensors) needs to be.

\section{Acknowledgement}

The research leading to these results has received funding from the European Commission under the FP7 Research Infrastructures project AIDA, grant agreement no. 262025. The information herein only reflects the views of its authors and not those of the European Commission and no warranty expressed or implied is made with regard to such information or its use.

\section{References}

[1] T. Aaltonen et al., Operational experience, improvements, and performance of the CDF Run II silicon vertex detector, NIM A 729 153-181 (2013).

[2] G. Barone, ATLAS silicon microstrip tracker operation and performance, NIM A 732, 57-60 (2013).

[3] Merkel, P. CMS tracker performance, NIM A 718, 339-341 (2013).

[4] F. Hartmann, Silicon tracking detectors in high-energy physics, NIM A 666, 25-46 (2012).

[5] L. Rossi and O. Brüning, High Luminosity Large Hadron Collider - A description for the European Strategy Preparatory Group, CERN-ATS-2012-236. 
[6] P. Weigell Recent results of the ATLAS upgrade planar pixel sensors R\&D project, NIM A 731, 177-182 (2013).

[7] A. Dierlamm, Silicon sensor developments for the CMS Tracker upgrade, Journal of Instrumentation 7, C01110 (2012).

[8] I. Mandić, Silicon sensors for HL-LHC tracking detectors, NIM A 732, 126-129 (2013).

[9] G. Casse et al., Enhanced efficiency of segmented silicon detectors of different thicknesses after proton irradiations up to $1 \times 10^{16} n_{\mathrm{eq}} \mathrm{cm}^{2}$, NIM A 624, 401-404 (2010).

[10] M. Moll, Recent progress of the RD50 Collaboration - Development of radiation tolerant tracking detectors, this issue.

[11] R. Eber, Investigations of new Sensor Designs and Development of an effective Radiation Damage Model for the Simulation of highly irradiated Silicon Particle Detectors, Karlsruhe Institute of Technology, PhD thesis, IEKP-KA/2013-27 (2013).

[12] R. Dalal et al., Simulations of Hadron Irradiated $n+p$-Si Strip Sensors Incorporating Bulk and Surface Damage, presented at 23rd RD50 Workshop, CERN, Nov. 2013 (on Indico the author is named Ranjeet Ranjeet)

[13] S. Kamada, Development of HPK $n$ in p Pixel Sensors for HL-LHC, presented at 9th HSTD, Hiroshima, 2013, to be published in NIM A.

[14] K.-H. Hoffmann, Campaign to identify the future CMS tracker baseline, NIM A 658, 30-35 (2011).

[15] M. Moll, Radiation Damage in Silicon Particle Detectors, Universität Hamburg, PhD thesis, DESY-THESIS-1999-040 (1999)

[16] P. Collins, Experience with Silicon Detectors at the DELPHI Experiment, LEP, proceedings presented at the 2nd International Symposium on Development and Application of Semiconductor Tracking Detectors, Hiroshima, Oct. 10-13, 1995,

delphiwww.cern.ch/ collinsp/paper94/hiroshima.ps.gz

[17] L. Snoj, G. Žerovnik, and A. Trkov, Computational analysis of irradiation facilities at the JSI TRIGA reactor, Applied Radiation and Isotopes 70, 483-488 (2012).

[18] I. Mandić et al., Bulk damage in DMILL npn bipolar transistors caused by thermal neutrons versus protons and fast neutrons, IEEE Transactions on Nuclear Science 51, 1752-1758 (2004).

[19] C. Betancourt et al., A charge collection study with dedicated RD50 charge multiplication sensors, NIM A 730, 62-65 (2013).

[20] L. Altan, Untersuchung zur Ladungsmultiplikation an hoch bestrahlten Siliziumstreifensensoren, Karlsruhe Institute of Technology, diploma thesis, IEKP-KA/2012-23 (2012).

[21] J. Harrison and A. Webber, Radiation damage in the LHCb VELO, NIM A 699, 193-197 (2013).

[22] K.-H. Hoffmann, Development of new Sensor Designs and Investigations on Radiation Hard Silicon Strip Sensors for the CMS Tracker Upgrade at the High Luminosity Large Hadron Collider, Karlsruhe Institute of Technology, PhD thesis, IEKP-KA/2013-1 (2013).

[23] M. Strelzyk, Design studies of n-in-p silicon strip sensors for the CMS tracker, Karlsruhe Institute of Technology, diploma thesis, IEKP-KA/2012-22 (2012).

[24] G. Hall, Conceptual study of a trigger module for the CMS Tracker at SLHC, NIM A 636, S201-S207 (2011).

[25] W. Treberspurg et al., Measuring doping profiles of silicon detectors with a custom-designed probe station, Journal of Instrumentation 7 P11009 (2012) and private communication. 